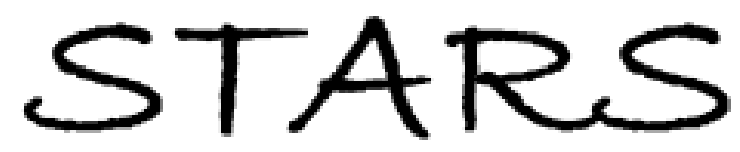

University of Central Florida

STARS

Faculty Bibliography 2000s

Faculty Bibliography

$1-1-2005$

\title{
Phase-only modulation using a normal-mode liquid-crystal gel
}

Hongwen Ren

University of Central Florida

Janet R. Wu

University of Central Florida

Shin-Tson Wu

University of Central Florida

Find similar works at: https://stars.library.ucf.edu/facultybib2000 University of Central Florida Libraries http://library.ucf.edu

This Article is brought to you for free and open access by the Faculty Bibliography at STARS. It has been accepted for inclusion in Faculty Bibliography 2000 s by an authorized administrator of STARS. For more information, please contactSTARS@ucf.edu.

\section{Recommended Citation}

Ren, Hongwen; Wu, Janet R.; and Wu, Shin-Tson, "Phase-only modulation using a normal-mode liquidcrystal gel" (2005). Faculty Bibliography 2000s. 5587.

https://stars.library.ucf.edu/facultybib2000/5587

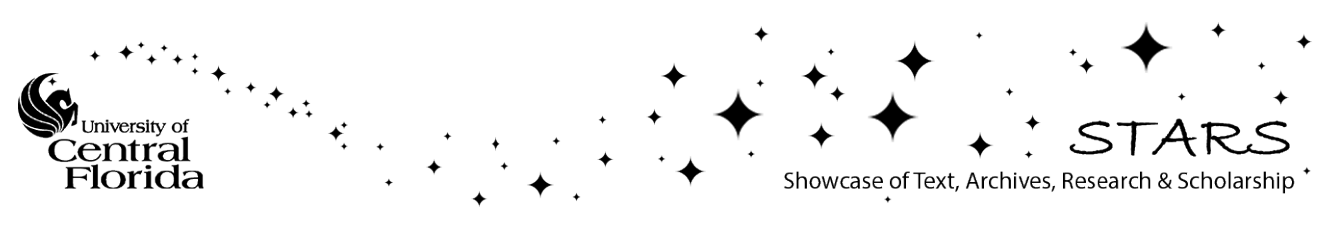




\section{Phase-only modulation using a normal-mode liquid-crystal gel}

Cite as: J. Appl. Phys. 98, 023104 (2005); https://doi.org/10.1063/1.1989436

Submitted: 28 January 2005 . Accepted: 06 June 2005. Published Online: 22 July 2005

Hongwen Ren, Janet R. Wu, and Shin-Tson Wu

ARTICLES YOU MAY BE INTERESTED IN

Polarization-independent phase modulation of a homeotropic liquid crystal gel

Applied Physics Letters 87, 191106 (2005); https://doi.org/10.1063/1.2126107

Fast-response and scattering-free polymer network liquid crystals for infrared light modulators

Applied Physics Letters 84, 1233 (2004); https://doi.org/10.1063/1.1649816

A polarization independent liquid crystal phase modulation adopting surface pinning effect of polymer dispersed liquid crystals

Journal of Applied Physics 110, 114516 (2011); https://doi.org/10.1063/1.3666053

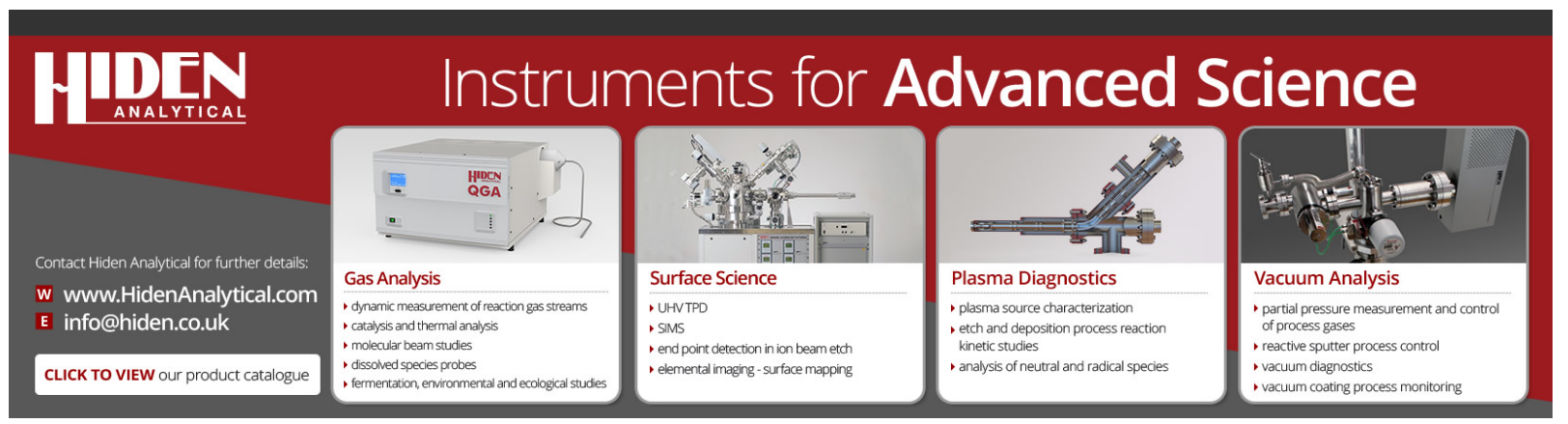




\title{
Phase-only modulation using a normal-mode liquid-crystal gel
}

\author{
Hongwen Ren, Janet R. Wu, and Shin-Tson $\mathrm{Wu}^{\mathrm{a})}$ \\ College of Optics and Photonics, University of Central Florida, Orlando, Florida 32816
}

(Received 28 January 2005; accepted 6 June 2005; published online 22 July 2005)

\begin{abstract}
A normal-mode liquid-crystal (LC) gel for phase-only modulation is demonstrated. In the absence of an electric field, the gel has negligible light scattering. Upon application of an electric field, the gel becomes highly transparent and exhibits phase modulation capability. For a $20-\mu \mathrm{m}$ transmissive E48 LC gel, the phase shift reaches $0.55 \pi$ at $\lambda=633 \mathrm{~nm}$ and the response time is $\sim 2 \mathrm{~ms}$. Although the phase change is not very large, it is sufficient for making adaptive microphotonic devices. Such a normal-mode LC gel-based phase modulator is free from scattering and hysteresis and has a fast response time and good long-term operation stability. (C) 2005 American Institute of Physics.
\end{abstract} [DOI: $10.1063 / 1.1989436]$

\section{INTRODUCTION}

Phase-only modulation is particularly desirable for laser beam steering, ${ }^{1}$ tunable-focus lens, ${ }^{2,3}$ and spatial light modulators. ${ }^{4}$ To achieve phase-only modulation, several approaches such as homogeneous nematic liquid crystal (LC), twisted-nematic (TN) LC, ${ }^{6-8}$ nanosized polymer-dispersed liquid crystal (nano-PDLC), ${ }^{9-11}$ polymer network liquid crystal (PNLC), ${ }^{12}$ sheared PNLC (or stressed LC), ${ }^{13,14}$ and conventional PDLC ${ }^{15}$ have been investigated. Each approach has its own merits and demerits. The homogeneous and TN cells possess a large phase change and low operating voltage, but their response times are relatively slow $(\sim 30 \mathrm{~ms}$ for a 5 - $\mu \mathrm{m}$ cell gap). The nano-PDLC is polarization independent and has submillisecond response time; however, its phase change is small and the required operating voltage is quite high $(10 \mathrm{~V} / \mu \mathrm{m})$. The sheared PNLC exhibits a large phase shift and a fast response time; however, its electro-optic properties are polarization sensitive and the required operating voltage is relatively high. The PDLC-based phase-only modulator is polarization independent and has a fast response time, but its phase shift is very small because the residual phase occurs above the saturated voltage. ${ }^{15}$ To develop phase-only modulators with a large phase shift, a low operating voltage, and a fast response time is a challenging task.

Polymer stabilization is a useful method for improving the response time of a LC-based phase modulator. A polymer-stabilized liquid crystal (also known as LC gel) ${ }^{16,17}$ usually contains a few (5-10) weight percent of polymer in a LC host. Most of the LC gels reported previously belong to the reversed mode; i.e., in the absence of an electric field the polymer-stabilized LC directors are aligned along the substrate surfaces. The reversed-mode LC gel exhibits a fast response time with a relatively low operating voltage. However, the drawbacks are light scattering in the visible wavelength, hysteresis, and long-term operation instability.

In this paper, we demonstrate a normal-mode LC gel for phase modulation. The normal-mode LC gel represents a compromised approach in terms of phase change, response

${ }^{a)}$ Electronic mail: swu@mail.ucf.edu time, and operating voltage. Its overall performance is between a homogeneous cell and a nano-PDLC. In a normalmode gel, the LC directors are stabilized by a polymer network in the absence of an electric field and present a quasihomeotropic alignment. ${ }^{18}$ Light scattering is weak in the voltage-off state. Upon application of an electric field, the gel becomes highly transparent and exhibits a phase modulation capability. Although the tunable phase shift is not too large, it is sufficient for making adaptive microphotonic devices. The normal-mode LC gel-based phase modulator is free from light scattering and hysteresis and has a fast response time and a good long-term operation stability.

\section{EXPERIMENT}

\section{A. Sample preparation}

To prepare a normal-mode anisotropic LC gel, we mixed 3 wt $\%$ of a rodlike photocurable monomer (bisphenol-Adimethacrylate) with $0.5 \mathrm{wt} \%$ photoinitiator in a nematic LC host (Merck E48, $n_{0}=1.523$, and $\Delta n=0.231$ ). The LC/ monomer mixture was injected to a homogeneous cell and then cured by ultraviolet light under a biased voltage. The inner surfaces of the indium tin oxide (ITO) glass substrates were overcoated with a thin polyimide layer and buffed in antiparallel directions. The filled cell was exposed to UV light $\left(\lambda \sim 365 \mathrm{~nm}, I \sim 10 \mathrm{~mW} / \mathrm{cm}^{2}\right)$ at room temperature $(T$ $\sim 21{ }^{\circ} \mathrm{C}$ ) with a $30-\mathrm{V}_{\mathrm{rms}}$ biased voltage. Two cells with gaps $d=10$ and $20 \mu \mathrm{m}$ were fabricated. The UV curing time for each cell was $\sim 40 \mathrm{~min}$.

Figures 1(a)-1(c) show the schematic diagrams illustrating the molecular arrangements in the voltage-off and two voltage-on states $\left(V_{2}>V_{1}\right)$ of the normal-mode LC gel. In Fig. 1(a) where $V=0$, the orientation of the formed polymer networks is almost perpendicular to the substrate surfaces. The LC directors inside the domains are bounded by the polymer networks but also influenced by the buffed polyimide alignment layers. Some LC domains are randomly oriented so that a weak scattering is present in the voltage-off state. As the applied voltage increases, the LC directors are reoriented along the electric-field direction. As a result, the LC gel becomes highly transparent, as shown in Fig. 1(b). Further increase in voltage would cause more LC directors to 


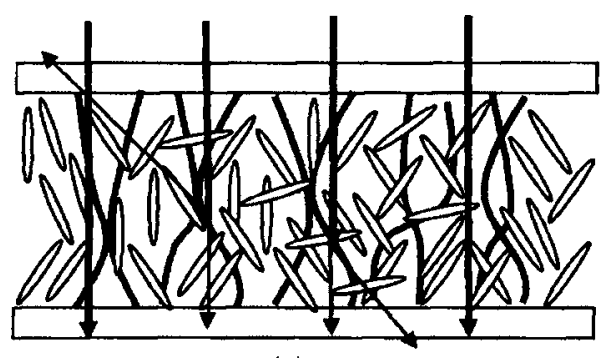

(a)

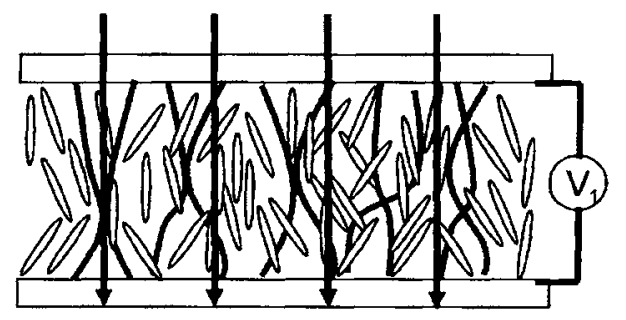

(b)

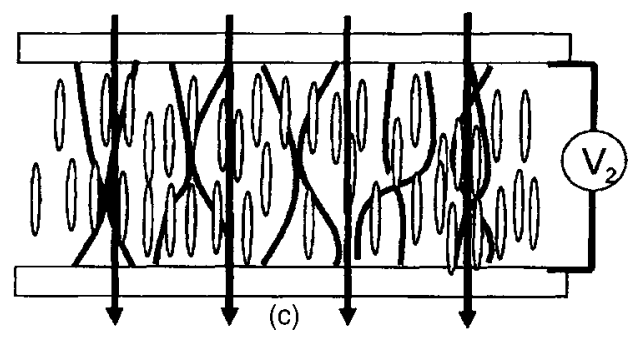

FIG. 1. Schematic diagrams of polymer network and LC director orientations of a normal-mode LC gel: (a) $V=0$, (b) $V_{1}$, and (c) $V_{2}>V_{1}$.

align along the electric-field direction, as shown in Fig. 1(c). From Figs. 1(b) and 1(c), the phase-only modulation is realized.

\section{B. Voltage-dependent transmittance}

The electro-optic properties of the LC gels were characterized by measuring their transmittance using an unpolarized $\mathrm{He}-\mathrm{Ne}$ laser beam $(\lambda=633 \mathrm{~nm})$. The transmitted light was measured by a photodiode detector which was placed at $\sim 30 \mathrm{~cm}$ behind the sample, corresponding to a $2^{\circ}$ collection angle. The response time of the $\mathrm{LC}$ gels was recorded by a digital oscilloscope. A computer controlled LABVIEW data acquisition system was used for driving the sample and recording the light transmittance.

Figures 2(a) and 2(b) plot the voltage-dependent transmittance of the 10- and 20- $\mu \mathrm{m}$ LC gels, respectively. An unpolarized $\mathrm{He}-\mathrm{Ne}$ laser beam was used for these measurements. From Fig. 2(a), the $10-\mu \mathrm{m}$ LC gel is highly transparent even at $V=0$. As the voltage increases, the transmittance remains at $\sim 90 \%$. For reference, the maximum transmittance is $92 \%$ due to the interface reflections between the glass substrates and the air. On the other hand, the 20- $\mu \mathrm{m}$ LC gel has a weak light scattering at $V=0$, as shown in Fig. 2(b), because of the thick cell gap. However, the transmittance still reaches $90 \%$ in the $V>10 \mathrm{~V}_{\text {rms }}$ region. The hysteresis of the voltage-dependent transmittance of each cell was also measured, as shown in Figs. 2(a) and 2(b), respec-
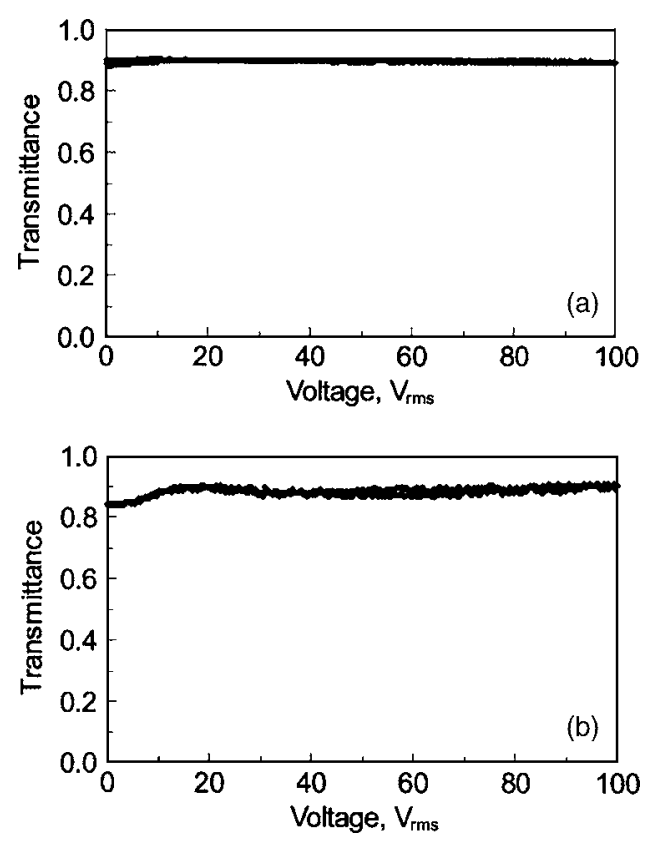

FIG. 2. Voltage-dependent transmittance of two normal-mode E48 LC gels: (a) $d=10 \mu \mathrm{m}$ and (b) $d=20 \mu \mathrm{m}$. An unpolarized He-Ne laser was used for these measurements.

tively. In each figure, the forward and backward curves overlap very well. This means the hysteresis in our normal-mode LC gels is completely suppressed.

To examine whether the LC gels exhibit a phase modulation capability, we measured their transmittance at $\lambda$ $=633 \mathrm{~nm}$ between parallel and crossed polarizers. In these measurements, the rubbing direction of the gel cells was orientated at $45^{\circ}$ with respect to the polarization axis of the polarizer. Results are plotted in Figs. 3(a) and 3(b) for the $10-$ and $20-\mu \mathrm{m}$ gels, respectively. In both figures, the transmittance increases for the parallel polarizer configuration but
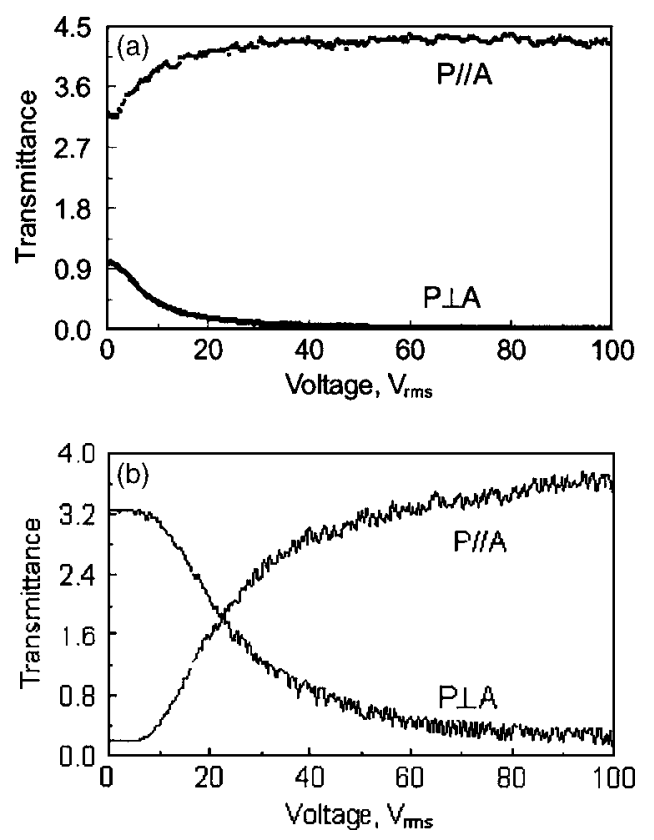

FIG. 3. Voltage-dependent transmittance of the E48 LC gels between parallel and crossed polarizers: (a) $d=10 \mu \mathrm{m}$ and (b) $d=20 \mu \mathrm{m}$. $f=1 \mathrm{kHz}, \lambda$ $=633 \mathrm{~nm}$, and $T \sim 21^{\circ} \mathrm{C}$. 


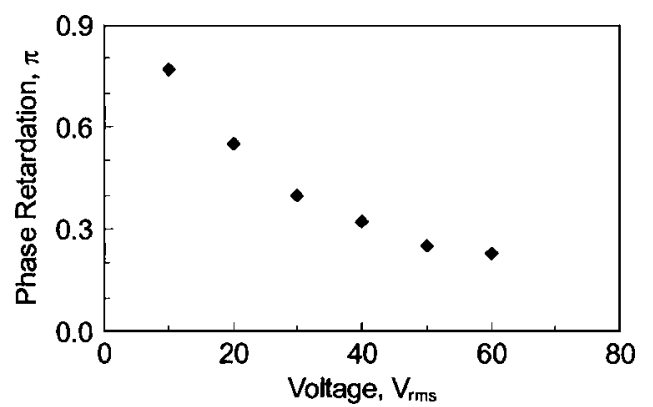

FIG. 4. Measured phase shift of the 20- $\mu \mathrm{m}$ E48 LC gel at different voltages.

decreases gradually for the crossed polarizers. These results imply that the normal-mode LC gel can indeed produce a phase-only modulation. The phase change at a given voltage can be calculated by taking the ratio of the transmitted intensities. ${ }^{19}$ The achievable phase change of the $10-\mu \mathrm{m}$ E48 LC gel from 0 to $60 \mathrm{~V}_{\text {rms }}$ is $\delta \sim 0.25 \pi$, which is somewhat too small for most of the applications. Therefore, we focus the discussions on the $20-\mu \mathrm{m}$ LC gel.

Figure 4 plots the voltage-dependent phase change of the 20- $\mu \mathrm{m}$ E48 LC gel at $\lambda=633 \mathrm{~nm}$ and $T \sim 21{ }^{\circ} \mathrm{C}$. At $V=0$, the thick sample has a weak light scattering. Thus, we measured the phase behavior starting from $V=10 \mathrm{~V}_{\mathrm{rms}}$. As the voltage increases, the residual phase decreases gradually because the LC directors inside the domains are reoriented by the electric field, leading to a decreased birefringence. From 10 to $60 \mathrm{~V}_{\text {rms }}$, the phase change is $\delta \sim 0.55 \pi$, which is about twice that of the $10-\mu \mathrm{m}$ gel. For the $20-\mu \mathrm{m}$ LC gel, the $60-\mathrm{V}_{\text {rms }}$ applied voltage corresponds to $3 \mathrm{~V} / \mu \mathrm{m}$. This electric-field strength is $\sim 3 \mathrm{X}$ smaller than that of the nanoPDLC devices. The low operating voltage of our normalmode LC gel originates from its low polymer concentration. If we operate the gel in reflective mode, the phase change will be doubled.

Such a gel's phase change is polarization dependent. To realize the phase modulation, the polarization of the incident light should be parallel to the substrate rubbing direction of the homogeneous cell. Although the obtainable phase shift is not too large, it is still sufficient for making microphotonic devices, such as microlens and microprisms. To increase the phase shift, we could either enlarge the LC cell gap or use a high-birefringence LC material. ${ }^{20}$ The latter is preferred because increasing cell gap would lead to a high operating voltage.

\section{Response time}

Response time is a very important parameter for almost every LC device. We measured the response time of the $20-\mu \mathrm{m}$ LC gel using square voltage bursts at $f=1 \mathrm{kHz}$ between 10 and $100 \mathrm{~V}_{\text {rms }}$. Results are shown in Fig. 5. The measured rise time is $\tau_{\text {rise }} \sim 0.35 \mathrm{~ms}$ and the decay time is $\tau_{\text {decay }} \sim 2 \mathrm{~ms}$ at room temperature. The rise $\left(\tau_{\text {rise }}\right)$ and decay $\left(\tau_{\text {decay }}\right)$ times of a nematic LC device are dependent on the cell gap $(d)$, rotational viscosity $\left(\gamma_{1}\right)$, elastic constant $(K)$, threshold voltage $\left(V_{\text {th }}\right)$, bias voltage $\left(V_{b}\right)$, and applied voltage $(V)$ as follows: ${ }^{21}$
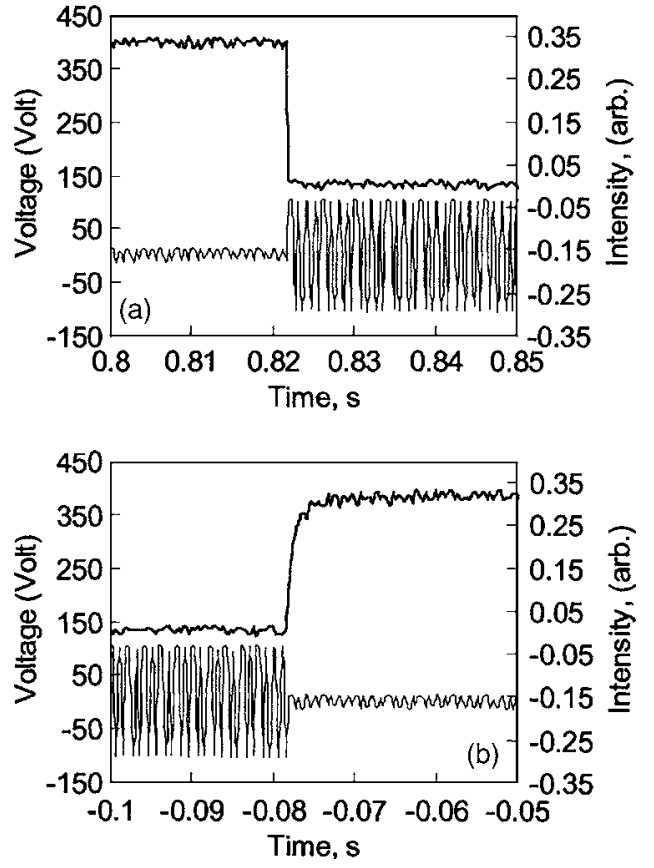

FIG. 5. The measured response time of the $20-\mu \mathrm{m}$ LC gel: (a) $\tau_{\text {rise }}$ $\sim 0.35 \mathrm{~ms}$ and (b) $\tau_{\text {decay }} \sim 2 \mathrm{~ms}$ at $T \sim 21{ }^{\circ} \mathrm{C}$. The lower trace in each figure represents the applied voltage bursts $(f=1 \mathrm{kHz})$, and the upper trace represents the optical signals.

$$
\begin{aligned}
& \tau_{\text {rise }}=\frac{\gamma_{1} d^{2}}{K \pi^{2}\left(V / V_{\text {th }}-1\right)}, \\
& \tau_{\text {decay }}=\frac{\gamma_{1} d^{2}}{K \pi^{2}\left|\left(V_{b} / V_{\text {th }}\right)^{2}-1\right|} .
\end{aligned}
$$

A normal-mode LC gel exhibits a lower $V_{\text {th }}$ than its corresponding reversed-mode gel due to the high tilt angle of the LC directors, i.e., quasihomeotropic alignment. From Eq. (1), a large $V / V_{\text {th }}$ leads to a fast rise time. For phase modulation, the LC gel is biased at a $V_{b}$ which is much higher than $V_{\text {th }}$. From Eq. (2), a large $V_{b} / V_{\text {th }}$ helps to reduce decay time. Unlike a pure nematic LC cell, the LC domains in a gel are surrounded by polymer networks. Therefore, the parameter $d$ in Eqs. (1) and (2) should represent the average LC domain size rather than the cell gap. For a given LC and monomer system, the domain size is mainly controlled by the monomer concentration. A smaller LC domain size (higher monomer concentration) is favorable for reducing response time, but the operating voltage would be higher. A low-viscosity LC mixture is always helpful for achieving a fast response time.

\section{CONCLUSION}

We have demonstrated a normal-mode LC gel for phaseonly applications. Such a phase modulation is free from light scattering and hysteresis. Its response time is reasonably fast $\left(\sim 2 \mathrm{~ms}\right.$ at $\left.T \sim 21^{\circ} \mathrm{C}\right)$, and its operation stability is excellent. Although the obtainable phase change is not too large, it is still sufficient for several microphotonic devices, such as tunable-focus microlens arrays. In comparison to a homogeneous or TN cell, the normal-mode LC gel possesses a 
smaller phase shift, but its response time is much faster. On the other hand, in comparison to a nano-PDLC system, the normal-mode LC gel has a larger phase change and a much lower operating voltage, although its response time is slower. In comparison to the reversed-mode LC gel, the normalmode LC gel is free from scattering and hysteresis, and its long-term operation stability is much better.

\section{ACKNOWLEDGMENTS}

The authors are indebted to Yi-Hsin Lin and Dr. YunHsing Fan for their technical assistance. This work was supported by Raytheon Electronic Systems Company.

${ }^{1}$ P. F. McManamon et al., Proc. IEEE 84, 268 (1996).

${ }^{2}$ G. D. Love, Appl. Opt. 32, 2222 (1993).

${ }^{3}$ H. Ren, Y. H. Fan, S. Gauza, and S. T. Wu, Appl. Phys. Lett. 84, 4789 (2004).

${ }^{4}$ U. Efron, Spatial Light Modulators (Dekker, New York, 1995).

${ }^{5}$ V. Freedericksz and V. Zolina, Trans. Faraday Soc. 29, 919 (1933).

${ }^{6}$ N. Konforti, E. Marom, and S. T. Wu, Opt. Lett. 13, 251 (1988).
${ }^{7}$ J. S. Patel, Appl. Phys. Lett. 58, 1314 (1991).

${ }^{8}$ Y. Huang, T. X. Wu, and S. T. Wu, J. Appl. Phys. 93, 2490 (2003).

${ }^{9}$ R. L. Sutherland, V. P. Tondiglia, L. V. Natarajan, T. J. Bunning, and W. W. Adams, Appl. Phys. Lett. 64, 1074 (1994).

${ }^{10}$ A. Y. G. Fuh, M. S. Tsai, L. J. Huang, and T. C. Liu, Appl. Phys. Lett. 74, 2572 (1999).

${ }^{11}$ D. E. Lucchetta, R. Karapinar, A. Manni, and F. Simoni, J. Appl. Phys. 91, 6060 (2002)

${ }^{12}$ Y. H. Fan, Y. H. Lin, H. Ren, and S. T. Wu, Appl. Phys. Lett. 84, 1233 (2004).

${ }^{13}$ Y. H. Wu, Y. H. Lin, Y. Q. Lu, H. Ren, Y. H. Fan, J. R. Wu, and S. T. Wu, Opt. Express 12, 6377 (2004).

${ }^{14}$ J. L. West, G. Zhang, A. Glushchenko, and Y. Reznikov, Appl. Phys. Lett. 86, 031111 (2005).

${ }^{15}$ H. Ren, Y. H. Lin, Y. H. Fan, and S. T. Wu, Appl. Phys. Lett. 86, 141110 (2005).

${ }^{16}$ R. A. M. Hikmet, J. Appl. Phys. 68, 4406 (1990).

${ }^{17}$ R. H. M. Hikmet, Phys. Rev. E 51, 5824 (1995).

${ }^{18}$ Y. H. Fan, H. Ren, and S. T. Wu, Appl. Phys. Lett. 82, 2945 (2003).

${ }^{19}$ S. T. Wu, U. Efron, and L. D. Hess, Appl. Opt. 23, 3911 (1984).

${ }^{20}$ S. Gauza, H. Wang, C.-H. Wen, S.-T. Wu, A. J. Seed, and R. Dabrowski, Jpn. J. Appl. Phys., Part 1 42, 3463 (2003).

${ }^{21}$ S. T. Wu and D. K. Yang, Reflective Liquid Crystal Displays (Wiley, New York, 2001). 